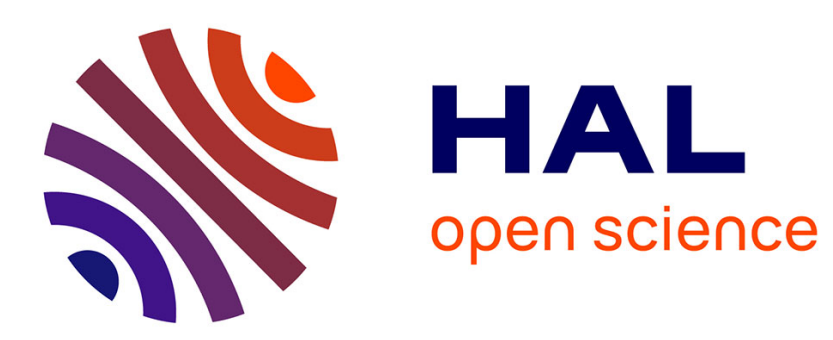

\title{
A Simplified Backstepping Design for 3D Time-based Aircraft Relative Guidance
}

Thierry Miquel, Felix Mora-Camino, Francis Casaux, Jean-Marc Loscos

\section{To cite this version:}

Thierry Miquel, Felix Mora-Camino, Francis Casaux, Jean-Marc Loscos. A Simplified Backstepping Design for 3D Time-based Aircraft Relative Guidance. AIAA GNC 2004, AIAA Guidance, Navigation and Control Conference and Exhibit, Aug 2004, Providence, United States. pp xxxx, 10.2514/6.20044993. hal-00998749

\section{HAL Id: hal-00998749 \\ https://hal-enac.archives-ouvertes.fr/hal-00998749}

Submitted on 2 Jun 2014

HAL is a multi-disciplinary open access archive for the deposit and dissemination of scientific research documents, whether they are published or not. The documents may come from teaching and research institutions in France or abroad, or from public or private research centers.
L'archive ouverte pluridisciplinaire $\mathbf{H A L}$, est destinée au dépôt et à la diffusion de documents scientifiques de niveau recherche, publiés ou non, émanant des établissements d'enseignement et de recherche français ou étrangers, des laboratoires publics ou privés. 


\title{
A simplified backstepping design for 3D time based aircraft relative guidance
}

\author{
Thierry Miquel* \\ LAAS du CNRS and Centre d'Etudes de la Navigation Aérienne, Toulouse, FRANCE \\ Félix Mora-Camino ${ }^{\dagger}$ \\ LAAS du CNRS and Ecole Nationale de l'Aviation Civile, Toulouse, FRANCE \\ and \\ Francis Casaux ${ }^{\ddagger}$, Jean-Marc Loscos $\$$ \\ Centre d'Etudes de la Navigation Aérienne, Toulouse, FRANCE
}

\begin{abstract}
The delegation to the flight crew of some tasks currently performed by air traffic controllers provides new perspectives to potentially increase air traffic control capacity. The objective of this communication is to provide technical insight into the airborne devices and algorithms which may be used to automatically perform merging and station keeping operations. Indeed, these maneuvers in the field of civil aviation seem difficult to be performed manually and may result in this case in an increase of the flight crew workload. Nevertheless new automated functions onboard aircraft could help to overcome this limitation. This paper investigates the design of a new autopilot mode dedicated to merging and station keeping maneuvers behind a leading aircraft. The proposed relative guidance law considers a 3-D relative motion, including constant wind and lateral, longitudinal and vertical control. It is based on vectorial backstepping and takes advantage of the skewsymmetric matrix which appears in the relative motion equations. An alternative 'simplified' design based on a matrix form of the Young's inequality is also presented in order to simplify the computation of the guidance law. Then, an illustrative example is discussed and conclusions are raised.
\end{abstract}

\section{Introduction}

$\mathrm{T}$ HIS paper investigates the design of a new autopilot mode dedicated to automatic merging and station keeping operations behind a leading aircraft. From an operational point of view, and assuming normal operations, automatic merging and station keeping operations relieve the air traffic controller of providing time consuming radar vectoring instructions to the trailing aircraft once the flight crew has accepted the relative guidance clearance. Thus, the expected benefit of such new capabilities onboard aircraft is an increase of air traffic controller availability, which could result in increased air traffic capacity and/or safety ${ }^{1}$. Enhancement of flight crew airborne traffic situational awareness with associated safety benefits is also expected.

The feasibility of such a relative guidance device is based on the ability of each aircraft to broadcast and receive suitable navigation data thanks to Automatic Dependent Surveillance-Broadcast (ADS-B) ${ }^{2}$. Among those navigation data, identification, position, altitude, groundspeed, vertical speed and track angle are of interest for the design of the relative guidance control law.

\footnotetext{
* Research engineer, Airborne Surveillance, Collision Avoidance and Separation Department, miquel@cena.fr

$\dagger$ Senior Researcher at LAAS du CNRS, Professor of Automatic Control and Avionics at Ecole Nationale de l'Aviation civile, Felix.MORA@enac.fr

¿CARE/ASAS manager \& AP1 PoC, casaux@cena.fr

${ }^{\S}$ Head of Airborne Surveillance, Collision Avoidance and Separation Department at CENA, loscos@cena.fr
} 
Despite a quite large literature dealing with aircraft relative guidance for Unmanned Air Vehicles (UAV) or military aircraft, research for civil aircraft in this field is still in its initial stage. Indeed, performances of such aircraft are more constrained than those of military aircraft or UAV. In addition, safety and passenger comfort are crucial issues. Previous work has concentrated only on the station keeping phase : in ${ }^{3}$ station keeping is performed manually by the flight deck, whereas in ${ }^{4}$ the authors consider a proportional, integral, and derivative (PID) control to control longitudinal station keeping. Very few papers concentrate on the automatic control of the merging maneuver before maintaining the desired position behind the leading aircraft. Indeed, the merging maneuver exhibits large nonlinearities which cannot be handled by linear control approaches. In ${ }^{5}$ and ${ }^{6}$ nonlinear control approaches have been presented where the separation objective is expressed in terms of distance.

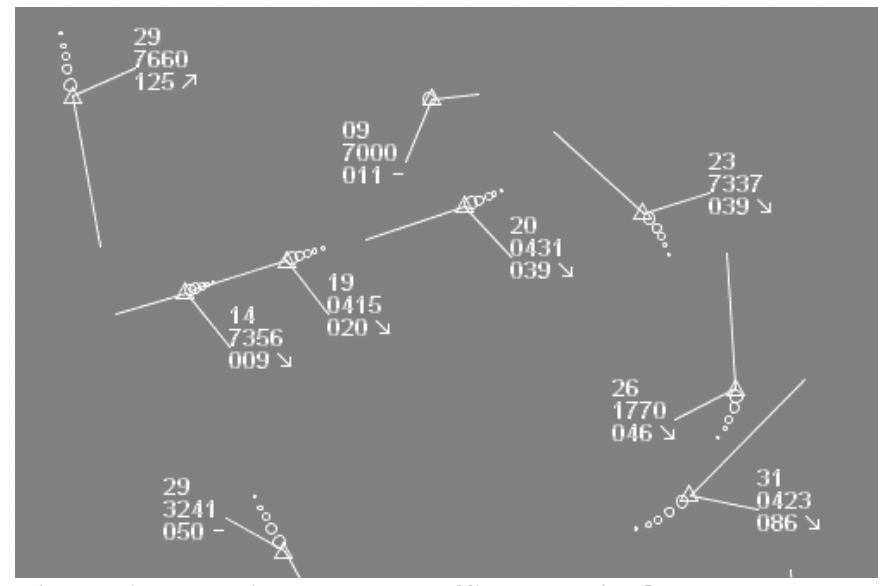

Figure 1: Terminal radar traffic at Paris-Orly

This paper investigates a time based separation

where the objective for the trailing aircraft is to track the position of the leading aircraft a few minutes earlier. The interest of such a criteria is that limiting constraints such as runway occupancy, wake vortex decay and human reactions are naturally expressed is terms of time ${ }^{7}$. On the other hand, as current civil aviation regulations set distance separation standard between aircraft in radar control airspace, the time based separation objective must be chosen so that the minimum distance separation standard is not violated.

The proposed automation of the relative guidance considers a 3-D relative motion, including constant wind and lateral, longitudinal and vertical control. It takes advantage of the cascaded structure of the flight dynamics through a recursive non-linear design, namely backstepping ${ }^{8}$. This is a quite new design methodology for construction of both feedback control laws and associated Lyapunov functions in a systematic manner.

The paper is organized as follows : in the preliminary section, the reference frame and the aircraft model are introduced. This leads to a nonlinear state space representation. The subsequent section presents the design of the controller through vectorial backstepping and makes use of the skew-symmetric matrix which appears in the relative motion equations. Then, an alternative 'simplified' design based on a matrix form of the Young's inequality is presented in order to decrease the complexity of the guidance law due to the "explosion of terms". Finally, an illustrative example taken from typical operations is provided in order to illustrate the approach, and conclusions are raised.

\section{Preliminaries}

\section{A. Relative position kinematics}

In the following, flat non rotating earth is considered as an inertial frame and standard atmosphere is assumed. In addition, and as depicted in Figure 2, the along track distance, denoted tk, is aligned with the trailing aircraft ground speed vector, whereas the cross track distance, denoted xtk, is the right handed positive distance from the trailing to the leading aircraft. Furthermore, $\chi$ stands for the track angle of the trailing aircraft, $G_{s}$ for its groundspeed and $\mathrm{V}$ for its true airspeed. Subscript $d$ is added for all variables related to the desired position : it represents the position and speed of the leading aircraft a few minutes earlier.

Assuming the same wind between the desired and the current position, it can be shown from Figure 2 that the relative motion kinematics between the current and the desired position are governed by the following equations :

$$
\left[\begin{array}{c}
\dot{t k} \\
\dot{x t k}
\end{array}\right]=\left[\begin{array}{cc}
0 & \dot{\chi} \\
-\dot{\chi} & 0
\end{array}\right]\left[\begin{array}{c}
t k \\
x t k
\end{array}\right]+\left[\begin{array}{c}
G_{s d} \cos \left(\chi_{d}-\chi\right)-G_{s} \\
G_{s d} \sin \left(\chi_{d}-\chi\right)
\end{array}\right]
$$


Furthermore, the relative motion in the vertical plane is the following, where $V_{z}$ stands for the vertical speed and $\gamma$ for the flight path angle :

$$
\Delta z=V_{z d}-\gamma \cdot V
$$

The track angle $\chi$ is the direction followed by the aircraft with respect to the local inertial frame, whereas the heading angle $\psi$ is the direction followed by the aircraft with respect to the air as far as the trailing aircraft is supposed to fly in a fully coordinated fashion, i.e. the side-slip angle is always zero. Denoting by $\psi_{w}$ the wind direction from where the wind is blowing (so that $\psi_{w}$ is zero when the wind is blowing from North) and by $W$ its velocity, the following relation holds :

$$
\left\{\begin{array}{l}
W_{x}=W \cdot \cos \left(\psi_{w}-\pi\right) \\
W_{y}=W \cdot \sin \left(\psi_{w}-\pi\right)
\end{array}\right.
$$

Furthermore, groundspeed and track angle are related to airspeed and heading angle through the following relations :

$$
\left\{\begin{array}{l}
G_{s}=\sqrt{V^{2}+W^{2}-2 \cdot V \cdot W \cdot \cos \left(\psi-\psi_{w}\right)} \\
\tan (\chi)=\frac{V \cdot \sin (\psi)-W \cdot \sin \left(\psi_{w}\right)}{V \cdot \cos (\psi)-W \cdot \cos \left(\psi_{w}\right)}
\end{array}\right.
$$

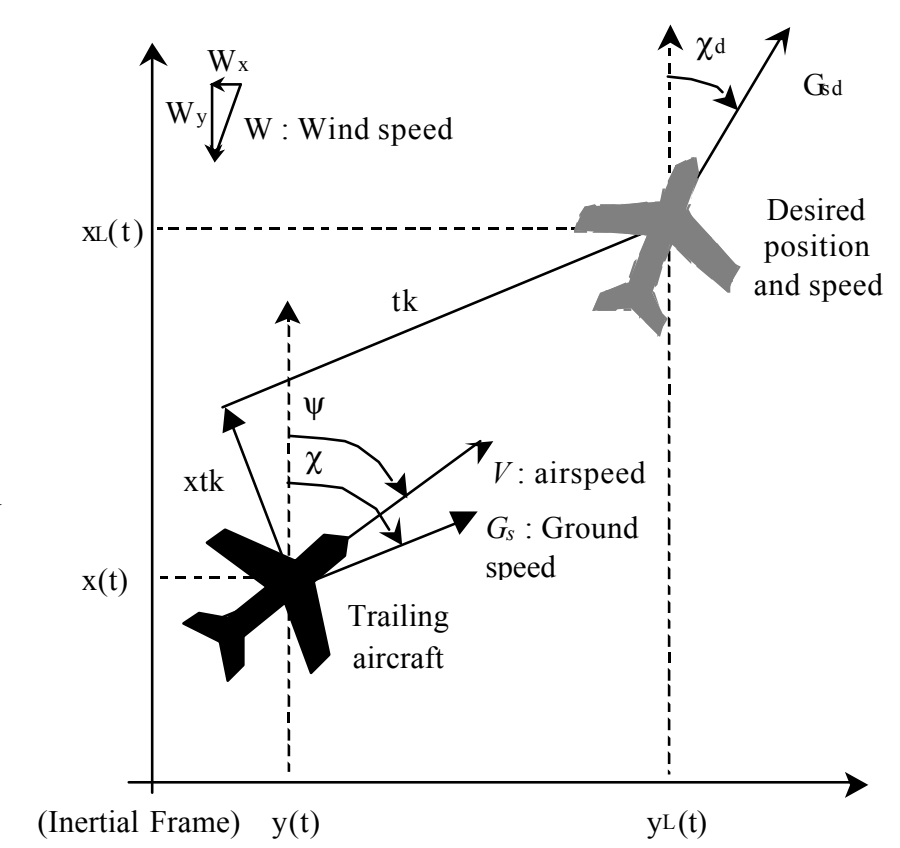

\section{B. Aircraft model}

The equations of motion used for the aircraft model are based on three-dimensional point mass differential equations, where $V$ is the true airspeed, $z$ the altitude, $\rho(z)$ the air mass density, $m$ the aircraft mass, $T_{0}$ the ratio between engine thrust and air mass density, $D_{0}(V, z)$ the ratio between drag and air mass density, $g$ the acceleration of gravity, $\gamma$ the flight path angle, $n_{z}$ the load factor and $\psi$ the heading angle.

$$
\left\{\begin{array}{l}
\dot{V}=\frac{\rho(z)}{m}\left(T_{0}-D_{0}(V, z)\right)-g \cdot \sin (\gamma) \\
\dot{\gamma}=\frac{g}{V}\left(n_{z}-1\right) \\
\dot{\psi} \approx \frac{g}{V} \cdot \varphi
\end{array}\right.
$$

Denoting $C_{x 0}$ the drag coefficient at zero lift, $C_{x i}$ the induce drag coefficient and $S$ the reference surface of the aircraft, the ratio $D_{0}(V, z)$ is computed thanks to the parabolic approximation of the polar curve, where the load factor has been assumed to remain close to 1 . Then :

$$
D_{0}(V, z) \approx \frac{1}{2} S V^{2} C_{x 0}+\frac{(m g)^{2}}{\frac{1}{2} S V^{2} \rho^{2}(z)} C_{x i}
$$

The ratio $T_{0}$ between engine thrust and air mass density, the load factor $n_{z}$ and the bank angle $\varphi$ are considered to be independent inputs which are adopted as controls.

\section{State space representation}

Gathering (1), (2) and (5) leads to the following state space representation of the relative guidance dynamics, where $\underline{u}$ denotes the control vector and $\underline{x}_{i}$ the state vector:

Where :

$$
\left\{\begin{array}{l}
\underline{\dot{x}}_{1}=\underline{a}\left(\underline{x}_{2}, \underline{u}^{\prime}\right) \cdot \underline{x}_{1}+\underline{b}\left(\underline{x}_{2}, \underline{x}_{2 d}\right) \\
\underline{\dot{x}}_{2}=\underline{c}\left(\underline{x}_{2}\right)+\underline{d}\left(\underline{x}_{2}\right) \cdot \underline{u}
\end{array}\right.
$$




$$
\left\{\begin{array}{l}
\underline{x}_{1}=\left[\begin{array}{lll}
t k & x t k & \Delta z
\end{array}\right]^{T} \\
\underline{x}_{2}=\left[\begin{array}{lll}
V & \gamma & \psi
\end{array}\right]^{T} \\
\underline{u}=\left[\begin{array}{lll}
T_{0} & n_{z} & \varphi
\end{array}\right]^{T}
\end{array}\right.
$$

And :

$$
\left\{\begin{array}{l}
\underline{a}\left(\underline{x}_{1}, \underline{u}\right)=\left[\begin{array}{ccc}
0 & \dot{\chi} & 0 \\
-\dot{\chi} & 0 & 0 \\
0 & 0 & 0
\end{array}\right] \\
\underline{b}\left(\underline{x}_{2}, \underline{x}_{2 d}\right)=\left[\begin{array}{c}
G_{s d} \cos \left(\chi_{d}-\chi\right)-G_{s} \\
G_{s d} \sin \left(\chi_{d}-\chi\right) \\
V_{z d}-\gamma \cdot V
\end{array}\right]
\end{array}\right.
$$

It shall be noticed from (4) that groundspeed $G_{s}$ and track angle $\chi$ are function of true airspeed $V$, heading angle $\psi$ and wind speed and direction, $V_{w}$ and $\psi_{w}$ respectively.

Furthermore, we have :

$$
\left\{\begin{array}{c}
\underline{c}\left(\underline{x}_{2}\right)=\left[\begin{array}{c}
-\rho(z) \cdot D_{0}(V, z) / m-g \cdot \sin (\gamma) \\
-g / V \\
0
\end{array}\right] \\
\underline{d}\left(\underline{x}_{2}\right)=\left[\begin{array}{ccc}
\rho(z) / m & 0 & 0 \\
0 & g / V & 0 \\
0 & 0 & g / V
\end{array}\right]
\end{array}\right.
$$

In this state space representation, the desired groundspeed $G_{s d}$, the desired track angle $\chi_{d}$ and the desired vertical speed $V_{z d}$ are exogenous varying parameters broadcasted through ADS-B. This representation does not take into account the dynamics of bank angle, load factor and throttle ratio, which are linked to actuators dynamics.

\section{Relative guidance controller}

\section{A. Backstepping design}

The design objective is to render the equilibrium point $\left(\underline{x}_{1}=\underline{0} ; \underline{x}_{2}=\underline{x}_{2 d}\right)$ globally asymptotically stable. Since the nonlinear system (7) consists of two state vectors $\underline{x}_{1}$ and $\underline{x}_{2}$ and taking into account the fact that the matrix $a\left(\underline{x}_{2}\right)$ is skew-symmetric, the vectorial backstepping technique can be applied by considering this system as two cascaded systems.

In a first step, the virtual control $\underline{b}\left(\underline{x}_{2}\right)$ is chosen in order to stabilize $\underline{x}_{1}$ around $\underline{0}$. The virtual control is chosen as follows, where $\Lambda_{1}$ denotes a positive definite feedback gain matrix (tuning parameter) and $\underline{z}_{2}\left(\underline{x}_{2}, \underline{x}_{2 d}\right)$ a new state variable :

$$
\underline{b}\left(\underline{x}_{2}, \underline{x}_{2 d}\right)=\underline{z}_{2}\left(\underline{x}_{2}, \underline{x}_{2 d}\right)-\Lambda_{1} \underline{x}_{1}
$$

A candidate Lyapunov function for the $\underline{x}_{1}$-system is :

$$
V_{1}=\frac{k_{1}}{2} \underline{x}_{1}^{T} \underline{x}_{1}
$$

Where $k_{1}>0$. Taking into account (11) and the fact that the matrix $\underline{a}\left(\underline{x}_{2}\right)$ is skew-symmetric, the time derivative of (12) is :

$$
\dot{V}_{1}=-k_{1} \underline{x}_{1}^{T} \Lambda_{1} \underline{x}_{1}+k_{1} \underline{x}_{1}^{T} \underline{z}_{2}\left(\underline{x}_{2}, \underline{x}_{2 d}\right)
$$

We now turn our attention on the $\underline{z}_{2}$-system.

In a second step, the dynamics of $\underline{z}_{2}$ is computed by time differentiation of (11). Taking into account (7) and assuming $\underline{x}_{2 d}$ constant leads to :

$$
\nabla \underline{b}\left(\underline{x}_{2}\right) \cdot\left(\underline{c}\left(\underline{x}_{2}\right)+\underline{d}\left(\underline{x}_{2}\right) \cdot \underline{u}\right)=\underline{\dot{z}}_{2}\left(\underline{x}_{2}, \underline{x}_{2 d}\right)-\Lambda_{1} \underline{\dot{x}}_{1}
$$

A candidate Lyapunov function for the $\left(\underline{x}_{1}-\underline{z}_{2}\right)$ system is :

$$
V_{2}=V_{1}+\frac{1}{2} \underline{z}_{2}^{T} \underline{z}_{2}
$$


Denoting $L_{\underline{d}} \underline{b}\left(\underline{x}_{2}\right) \hat{=} \nabla \underline{b}\left(\underline{x}_{2}\right) \underline{d}\left(\underline{x}_{2}\right)$ the Lie derivative of $\underline{b}\left(\underline{x}_{2}\right)$ along $\underline{d}\left(\underline{x}_{2}\right)$ and taking into account (14), the time derivative of (15) is :

$$
\left.\dot{V}_{2}=\dot{V}_{1}+\underline{z}_{2}^{T}\left(L_{\underline{c}} \underline{b}\left(\underline{x}_{2}\right)+L_{\underline{d}} \underline{b} \underline{x}_{2}\right) \underline{u}+\Lambda_{1} \underline{\dot{x}}_{1}\right)
$$

Taking into account (13) leads to the following :

$$
\left.\left.\dot{V}_{2}=-k_{1} \underline{x}_{1}^{T} \Lambda_{1} \underline{x}_{1}+k_{1} \underline{z}_{2}^{T} \underline{x}_{1}+\underline{z}_{2}^{T}\left(\Lambda_{1} \underline{x}_{1}+L_{\underline{c}} \underline{b}_{2} \underline{x}_{2}\right)+L_{\underline{d}} \underline{b}^{2} \underline{x}_{2}\right) \underline{u}\right)
$$

For the relative guidance studied in this paper, the matrix $\nabla \underline{b}\left(\underline{x}_{2}\right)$ has the following expression :

Where :

$$
\left.\nabla \underline{b}_{2}\right)=\left[\begin{array}{ccc}
A(z, V, \psi) & 0 & B(V, \psi) \\
C(z, V, \psi) & 0 & D(V, \psi) \\
-\gamma & -V & 0
\end{array}\right]
$$

$$
\left\{\begin{array}{l}
A(z, V, \psi)=G_{s d} \frac{\partial \chi}{\partial V} \sin \left(\chi_{d}-\chi\right)-\frac{\partial G_{s}}{\partial V} \\
B(V, \psi)=G_{s d} \frac{\partial \chi}{\partial \psi} \sin \left(\chi_{d}-\chi\right)-\frac{\partial G_{s}}{\partial \psi} \\
C(z, V, \psi)=-G_{s d} \frac{\partial \chi}{\partial V} \cos \left(\chi_{d}-\chi\right) \\
D(V, \psi)=-G_{s d} \frac{\partial \chi}{\partial \psi} \cos \left(\chi_{d}-\chi\right)
\end{array}\right.
$$

The product $A D-B C$ has the dimension of a speed and is given by :

$$
A D-B C=G_{s d}\left(\frac{\partial G_{s}}{\partial V} \cdot \frac{\partial \chi}{\partial \psi}-\frac{\partial G_{s}}{\partial \psi} \cdot \frac{\partial \chi}{\partial V}\right) \cos \left(\chi_{d}-\chi\right)
$$

The partial derivative $\partial G_{s} \partial V, \partial G_{s} \partial \psi, \partial \chi \partial \partial$ and $\partial \chi \partial \psi$ are computed from (4).

Note : if wind is not considered (i.e. $\chi=\psi, G_{s}=V$ ), the above expressions reduce to :

$$
\left\{\begin{array}{l}
A(z, V, \psi)=-1 \\
B(V, \psi)=G_{s d} \sin \left(\chi_{d}-\chi\right) \\
C(z, V, \psi)=0 \\
D(V, \psi)=-G_{s d} \cos \left(\chi_{d}-\chi\right)
\end{array}\right.
$$

The matrix $L_{\underline{d}} \underline{b}\left(\underline{x}_{2}\right) \hat{=} \nabla \underline{b}_{2}\left(\underline{x}_{2}\right) \underline{d}_{2}\left(\underline{x}_{2}\right)$ is given by :

$$
L_{\underline{d}} \underline{b}\left(\underline{x}_{2}\right)=\left[\begin{array}{ccc}
\frac{\rho(z)}{m} A(z, V, \psi) & 0 & \frac{g}{V} B(V, \psi) \\
\frac{\rho(z)}{m} C(z, V, \psi) & 0 & \frac{g}{V} D(V, \psi) \\
-\frac{\gamma \rho(z)}{m} & -g & 0
\end{array}\right]
$$

The key point of the control law design is that matrix $L_{\underline{d}} \underline{b}\left(\underline{x}_{2}\right)$ is invertible. Indeed, we have :

$$
\begin{aligned}
& \left(L_{\underline{d}} \underline{b}\left(\underline{x}_{2}\right)\right)^{-1}=\frac{m V}{g \rho(z)(A D-B C)}\left[\begin{array}{lll}
\underline{c}_{1} & \underline{c}_{2} & \underline{c}_{3}
\end{array}\right] \\
& \text { where }\left\{\begin{aligned}
& \underline{c}_{1}= {\left[\begin{array}{c}
g D(V, \psi) / V \\
-\gamma \rho(z) D(V, \psi) / m V \\
-\rho(z) C(z, V, \psi) / m
\end{array}\right] } \\
& \underline{c}_{2}= {\left[\begin{array}{c}
-g B(V, \psi) / V \\
\gamma \rho(z) B(V, \psi) / m V \\
\rho(z) A(z, V, \psi) / m
\end{array}\right] } \\
& 0 \\
& \underline{c}_{3}=\left[\begin{array}{c}
-\rho(z)(A D-B C) / m V \\
0
\end{array}\right]
\end{aligned}\right.
\end{aligned}
$$


Finally, control vector $\underline{u}$ is defined in order to regulate the virtual output $\underline{z}_{2}$ to zero.

A first alternative is to choose the control vector $\underline{u}$ as follows, where $\Lambda_{2}$ denotes a positive definite feedback gain matrix (tuning parameter) :

$$
\underline{u}=-\left(L_{\underline{d}} \underline{b}\left(\underline{x}_{2}\right)\right)^{-1} \cdot\left(k_{1} \underline{x}_{1}+\Lambda_{1} \underline{\dot{x}}_{1}+L_{\underline{c}} \underline{b}\left(\underline{x}_{2}\right)+\Lambda_{2} \underline{z}_{2}\right)
$$

So, the time derivative of the candidate Lyapunov function defined by (17) becomes :

$$
\dot{V}_{2}=-k_{1} \underline{x}_{1}^{T} \Lambda_{1} \underline{x}_{1}-\underline{z}_{2}^{T} \Lambda_{2} \underline{z}_{2}
$$

Hence, the $\left(\underline{x}_{1}-\underline{z}_{2}\right)$ system is stabilized.

An is sue with the proposed backstepping design is the complexity due to the "explosion of terms" arising from the calculation of the control vector : indeed, the control vector $\underline{u}$ needs the computation of five terms : $\left.\left(L_{\underline{d}} \underline{b}\left(\underline{x}_{2}\right)\right)^{-1}, k_{1} \underline{x}_{1}, \Lambda_{1} \underline{\dot{x}}_{1}, L_{\underline{c}} \underline{b}^{(\underline{x}} \underline{x}_{2}\right)$ and $\Lambda_{2} \underline{z}_{2}$.

In addition, as far as $\underline{\dot{x}}_{1}$ is a function of $\underline{u}$ as stated in the first equation of (7), the expression of the control vector $\underline{u}$ given by equation (24) is imp licit : in the case considered in this paper, $\underline{\dot{x}}_{1}$ is not measured and consequently the relation shall be manipulated in order to extract $\underline{u}$ as a function of $\underline{x}_{1}$ and $\underline{x}_{2}$.

\section{B. Alternative design}

An alternative design is developed in order to simplify the expression of the control vector $\underline{u}$ and to give an explicit formulation of it. This alternative design is based on the Young's inequality.

Taking into account (11) and the first equation of (7) into (17) leads to :

$$
\left.\left.\dot{V}_{2}=-k_{1} \underline{x}_{1}^{T} \Lambda_{1} \underline{x}_{1}+\underline{z}_{2}^{T}\left(k_{1} I+\Lambda_{1} \underline{a}\left(\underline{x}_{2}, \underline{u}\right)-\Lambda_{1}^{2}\right) \underline{x}_{1}+\underline{z}_{2}^{T}\left(\Lambda_{1} \underline{z}_{2}+L_{\underline{c}} \underline{b}_{2} \underline{x}_{2}\right)+L_{\underline{d}} \underline{b}_{2} \underline{x}_{2}\right) \underline{u}\right)
$$

Where matrix $I$ stands for identity matrix.

For the specific case studied in this paper, we will assume that matrix $\Lambda_{l}$ is diagonal :

$$
\Lambda_{1}=\operatorname{diag}\left\{\lambda_{11}, \lambda_{12}, \lambda_{13}\right\}
$$

As a consequence, the expression of $\left(k_{1} I+\Lambda_{1} \underline{a}\left(\underline{x}_{2}, \underline{u}\right)-\Lambda_{1}^{2}\right)$ is the following :

$$
\left(k_{1} I+\Lambda_{1} \underline{a}\left(\underline{x}_{2}, \underline{u}\right)-\Lambda_{1}^{2}\right)=\left[\begin{array}{ccc}
k_{1}-\lambda_{11}^{2} & \lambda_{11} \dot{\chi} & 0 \\
-\lambda_{12} \chi & k_{1}-\lambda_{12}^{2} & 0 \\
0 & 0 & k_{1}-\lambda_{13}^{2}
\end{array}\right]
$$

The scalar form of the Young's inequality is :

$$
x y \leq \frac{1}{2 d} x^{2}+\frac{d}{2} y^{2} \quad \forall d>0
$$

This inequality applied to (28) leads to :

$$
\underline{z}_{2}^{T}\left(k_{1} I+\Lambda_{1} \underline{a}\left(\underline{x}_{2}, \underline{u}\right)-\Lambda_{1}^{2}\right) \underline{x}_{1} \leq \underline{z}_{2}^{T}\left(\frac{D^{-1}}{2}+\frac{1}{2 k_{2}} I_{2}\right) \underline{z}_{2}+\underline{x}_{1}^{T}\left(\frac{D}{2} \mathrm{~K}_{1}^{2}+\frac{k_{2}}{2} \mathrm{~K}_{2}^{2}\right) \underline{x}_{1}
$$

Where :

$$
\left\{\begin{array}{l}
\mathrm{K}_{1}=\left[\begin{array}{ccc}
k_{1}-\lambda_{11}^{2} & 0 & 0 \\
0 & k_{1}-\lambda_{12}^{2} & 0 \\
0 & 0 & k_{1}-\lambda_{13}^{2}
\end{array}\right] \\
\mathrm{K}_{2}=\left[\begin{array}{ccc}
\lambda_{12} \dot{\chi} & 0 & 0 \\
0 & \lambda_{11} \dot{\chi} & 0 \\
0 & 0 & 0
\end{array}\right] \\
I_{2}=\left[\begin{array}{lll}
1 & 0 & 0 \\
0 & 1 & 0 \\
0 & 0 & 0
\end{array}\right]
\end{array}\right.
$$

Parameter $k_{2}$ is a positive design parameter and matrix $D$ is a design positive diagonal matrix of parameters $d_{i}$ :

$$
D=\operatorname{diag}\left\{d_{1}, d_{2}, d_{3}\right\}
$$

Therefore (26) implies :

$$
\dot{V}_{2} \leq-\underline{x}_{1}^{T}\left(k_{1} \Lambda_{1}-\frac{D}{2} \mathrm{~K}_{1}^{2}-\frac{k_{2}}{2} \mathrm{~K}_{2}^{2}\right) \underline{x}_{1}+\underline{z}_{2}^{T}\left(\frac{D^{-1}}{2}+\frac{1}{2 k_{2}} I_{2}\right) \underline{z}_{2}+\underline{z}_{2}^{T}\left(\Lambda_{1} \underline{z}_{2}+L_{\underline{c}} \underline{b}\left(\underline{x}_{2}\right)+L_{\underline{d}} \underline{b}\left(\underline{x}_{2}\right) \underline{u}\right)
$$


In order to stabilize the $\left(\underline{x}_{1}-\underline{z}_{2}\right)$ system, the control vector $\underline{u}$ is chosen as follows, where $\Lambda_{2}$ denotes a positive definite feedback gain matrix (tuning parameter) :

Thus (33) becomes :

$$
\underline{u}=-\left(L_{\underline{d}} \underline{b}\left(\underline{x}_{2}\right)\right)^{-1} \cdot\left(L_{\underline{c}} \underline{b}\left(\underline{x}_{2}\right)+\left(\Lambda_{1}+\Lambda_{2}\right) \underline{z}_{2}\right)
$$

$$
\dot{V}_{2} \leq-\underline{x}_{1}^{T}\left(k_{1} \Lambda_{1}-\frac{D}{2} \mathrm{~K}_{1}^{2}-\frac{k_{2}}{2} \mathrm{~K}_{2}^{2}\right) \underline{x}_{1}-\underline{z}_{2}^{T}\left(\Lambda_{2}-\frac{D^{-1}}{2}-\frac{1}{2 k_{2}} I_{2}\right) \underline{z}_{2}
$$

The time derivative of the candidate Lyapunov function $V_{2}$ can be made negative definite for a choice of $k_{1}, k_{2}$, $\Lambda_{1}, \Lambda_{2}$ and $D$ such that :

$$
\left\{\begin{array}{l}
k_{1} \Lambda_{1}-\frac{D}{2} \mathrm{~K}_{1}^{2}-\frac{k_{2}}{2} \mathrm{~K}_{2}^{2}>0 \\
\Lambda_{2}-\frac{D^{-1}}{2}-\frac{1}{2 k_{2}} I_{2}>0
\end{array}\right.
$$

Finally, the expression of the control vector $\underline{u}$ according to the initial state variables $\underline{x}_{1}$ and $\underline{x}_{2}$ is obtained by taking into account (11) into (34). This leads to :

$$
\underline{u}=-\left(L_{\underline{d}} \underline{b}\left(\underline{x}_{2}\right)\right)^{-1} \cdot\left(\left(L_{\underline{c}} \underline{b}\left(\underline{x}_{2}\right)+\left(\Lambda_{1}+\Lambda_{2}\right) \cdot\left(\Lambda_{1} \underline{x}_{1}+\underline{b}_{2}\left(\underline{x}_{2}, \underline{x}_{2 d}\right)\right)\right)\right.
$$

The control law (37) is 'simplified' compared to (24) in the sense that the time dependant terms $k_{1} \underline{x}_{1}$ and $\Lambda_{1} \underline{\dot{x}}_{1}$ have disappear through the bound defined by matrix inequalities (36).

The vector $L_{\underline{c}} \underline{b}\left(\underline{x}_{2}\right)$ has the following expression :

$$
L_{\underline{c}} \underline{b}_{2}\left(\underline{x}_{2}\right)=\left[\begin{array}{c}
0 \\
0 \\
g
\end{array}\right]-\left(\frac{\rho(z) \cdot D_{0}(V, z)}{m}+g \cdot \sin (\gamma)\left[\begin{array}{c}
A \\
C \\
-\gamma
\end{array}\right]\right.
$$

In the following, the matrix $\Lambda_{2}$ is chosen as a positive diagonal matrix of parameters $\lambda_{2 i}$ :

$$
\Lambda_{2}=\operatorname{diag}\left\{\lambda_{21}, \lambda_{22}, \lambda_{23}\right\}
$$

So :

$$
\begin{aligned}
& \left.\left(\Lambda_{1}+\Lambda_{2}\right) \cdot\left(\Lambda_{1} \underline{x}_{1}+\underline{b}_{(} \underline{x}_{2}, \underline{x}_{2 d}\right)\right)= \\
& {\left[\begin{array}{c}
\left(\lambda_{11}+\lambda_{21}\right)\left(\lambda_{11} t k+G_{s d} \cos \left(\chi_{d}-\chi\right)-G_{s}\right) \\
\left(\lambda_{12}+\lambda_{22}\right)\left(\lambda_{12} x t k+G_{s d} \sin \left(\chi_{d}-\chi\right)\right) \\
\left(\lambda_{13}+\lambda_{23}\right)\left(\lambda_{13} \Delta z+V_{z d}-\gamma \cdot V\right)
\end{array}\right]}
\end{aligned}
$$

Parameter $k_{1}$ has the dimension of $\sec ^{-2}, \Lambda_{1}$ and $\Lambda_{2}$ have the dimension of $\sec ^{-1}$ and $D$ and $k_{2}$ have the dimension of sec.

\section{Illustrative example}

\section{A. Scenario}

In this section, a scenario is designed in order to illustrate the properties of the control laws previously designed.

The leading aircraft trajectory starts at $x_{0}=0 \mathrm{NM}, \mathrm{y}_{0}=0 \mathrm{NM}$ and FL 100, with initial indicated airspeed of 220 kts and heading of 0 degrees. It is supposed to broadcast its data every second. No wind is considered in this example.

The leading aircraft is assumed to follow a typical arrival procedure, with two turns of 90 degrees. The first turn starts after about $8 \mathrm{~min}(495 \mathrm{sec})$ of flight, and the second turn starts after about $10 \mathrm{~min}(626 \mathrm{sec})$ of flight. The indicated airspeed decreases firstly towards $180 \mathrm{kts}$ and then towards $140 \mathrm{kts}$, and the flight level decreases towards 3000 feet after 2 min of flight.

The trailing aircraft trajectory starts at $\mathrm{x}_{0}=+10 \mathrm{NM}, \mathrm{y}_{0}=-7 \mathrm{NM}$ and FL 100, with initial indicated airspeed of 225 kts and heading of 330 degrees.

The simulation lasts $15 \mathrm{~min}(900 \mathrm{sec})$, and the requested time based separation for the trailing aircraft is constant and equal to $90 \mathrm{sec}$ behind the leading aircraft. The end of simulation is supposed to be the beginning of the final descent.

During the maneuver, the bank angle $\varphi$, the load factor $n_{z}$ and the indicated airspeed (denoted $C A S$ ) of the trailing aircraft are limited to the following 'passenger comfort' values : 


$$
\left\{\begin{array}{l}
-20 \mathrm{deg} . \leq \varphi \leq+20 \mathrm{deg} \\
0.94 \leq n_{z} \leq 1.06 \\
140 k t s \leq C A S \leq 250 k t s
\end{array}\right.
$$

In addition, longitudinal acceleration is limited to $0.05 \times \mathrm{g}$ and roll velocity to $5 \mathrm{deg}$./ $/ \mathrm{sec}$. In order to take into account the actuator dynamics, bank angle and load factor commands are filtered through a first order low pass filter with a time constant of 1.5 seconds, whereas throttle control is filtered through a first order low pass filter with a time constant of 5 seconds.

Following ${ }^{9}$, the drag coefficient at zero lift, denoted $C_{x 0}$, the induce drag coefficient, denoted $C_{x i}$, the reference surface of the aircraft, denoted $S$, and the maximum thrust available have been chosen as follows, which corresponds to a medium range turbojet aircraft :

$$
\left\{\begin{array}{l}
C_{x 0}=1.23 \cdot 10^{-2} \\
C_{x i}=6.056 \cdot 10^{-2} \\
S=825 \mathrm{ft}^{2} \\
\text { Thrust Max at sea level }=32000 \mathrm{lb}
\end{array}\right.
$$

Finally, the values of the constants defining the output vector dynamics have been chosen as follows:

$$
\left\{\begin{array} { l } 
{ \lambda _ { 1 1 } = 0 . 1 \mathrm { sec } ^ { - 1 } } \\
{ \lambda _ { 1 2 } = 0 . 1 \mathrm { sec } ^ { - 1 } } \\
{ \lambda _ { 1 3 } = 0 . 2 \mathrm { sec } ^ { - 1 } }
\end{array} \text { and } \left\{\begin{array}{l}
\lambda_{21}=0.12 \mathrm{sec}^{-1} \\
\lambda_{22}=0.12 \mathrm{sec}^{-1} \\
\lambda_{23}=0.3 \mathrm{sec}^{-1}
\end{array}\right.\right.
$$

Matrix inequalities (36) are satisfied by taking for example $\mathrm{k}_{1}=0.02 \mathrm{sec}^{-2}$ and $\mathrm{k}_{2}=9 \mathrm{sec}$.

\section{B. Results}

The achieved time based separation between the two aircraft is showed in Figure 3.

It has been computed off-line for each position of the leading aircraft as the difference between the time stamp of the leading aircraft position and the time stamp of the closest trailing aircraft position. As expected, the trailing aircraft moves towards the leading aircraft, with a delay of $90 \mathrm{sec}$. Moreover, despite two changes of 90 degrees in heading and $40 \mathrm{kts}$ in indicated airspeed (which start after 495 sec of flight), the achieved time based separation remains between $-1 \mathrm{sec}$ and $+2 \mathrm{sec}$ around the desired delay. The trailing aircraft is stabilized more than 3 minutes $(900-700 \mathrm{sec})$ before the end of the simulation.

Figure 4 and Figure 5 show the movements of the leading and trailing aircraft in the horizontal and in the vertical planes. The trailing aircraft makes slight overshoot during the turns, but no overshoot appears in the altitude tracking.

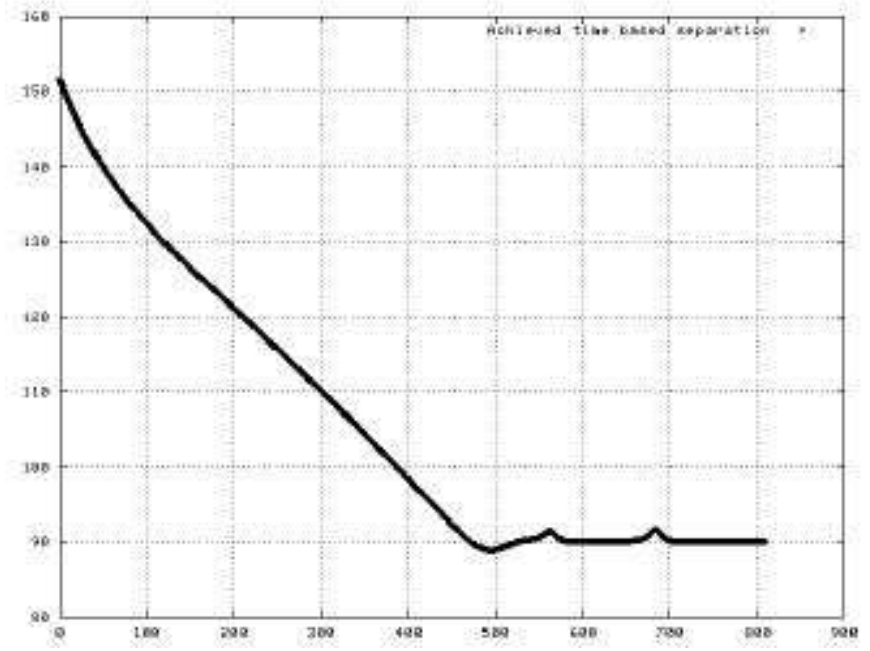

Figure 3: Achieved time based separation (in sec) between the two aircraft as a function of time (in sec) 


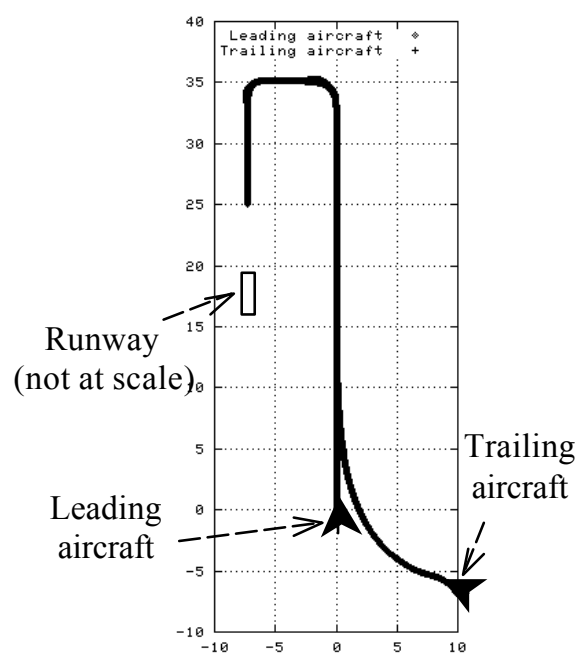

Figure 4: Leading aircraft and trailing aircraft trajectories in the horizontal plane (orthonormed axes in NM)

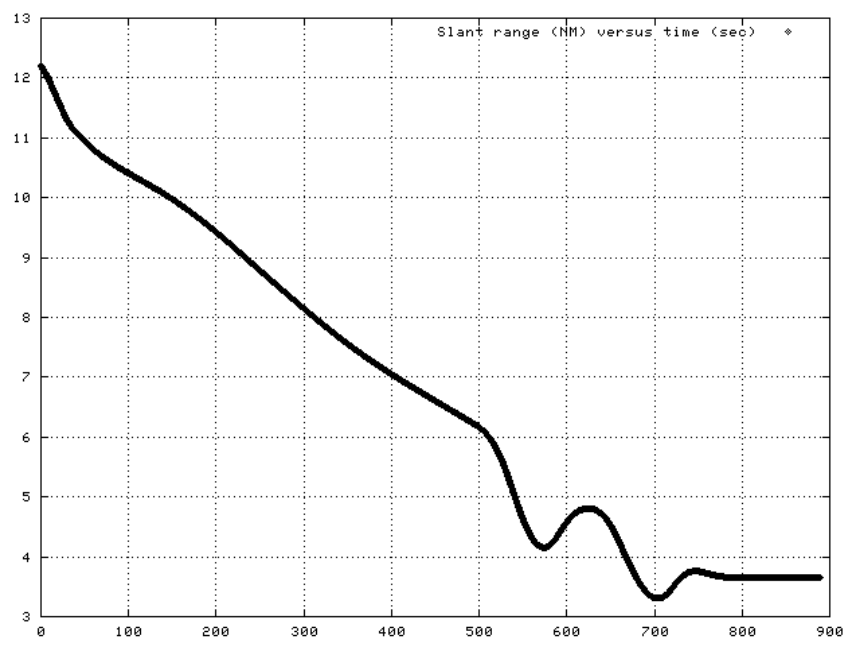

Figure 6: Actual slant range (in NM) between the two aircraft as a function of time (in sec)

Figure 6 shows the slant range between the two aircraft. The slant range always exceed $3 \mathrm{NM}$, which is encouraging from the distance separation standard compatibility point of view.

Finally, Figure 7 and Figure 8 show respectively the evolution of the indicated airspeed and throttle control. It appears that the throttle control is quite demanding.

\section{Conclusion}

In this paper, the design of a relative guidance controller to automatically move a trailing aircraft towards a leading aircraft and to maintain a constant time delay behind it has been considered. The design considers a 3-D relative motion, including constant wind and lateral, longitudinal and vertical control.

The proposed approach is based on vectorial backstepping. One of the key-point of such a design is the use of a skew-

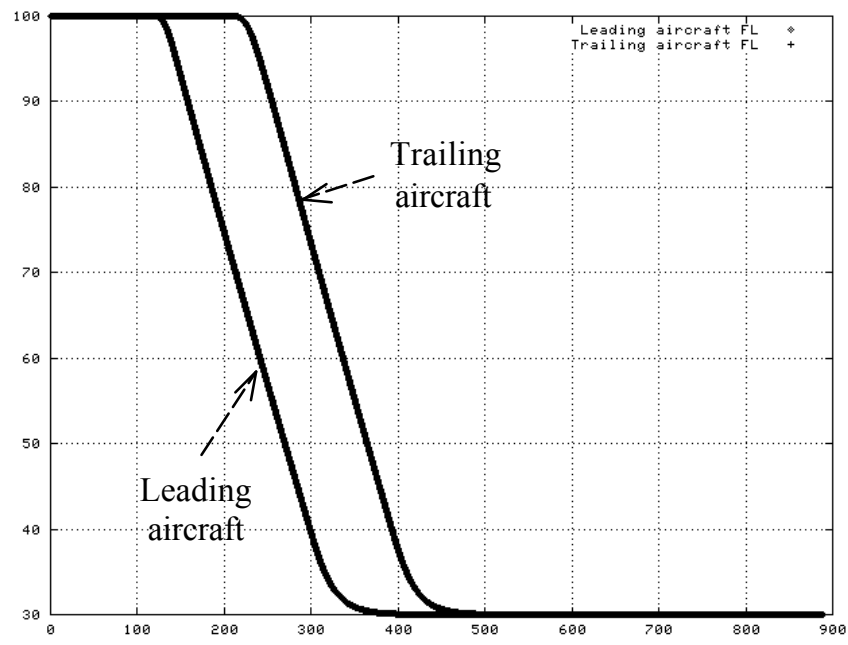

Figure 5: Leading aircraft and trailing aircraft flight levels (FL) versus time (sec)

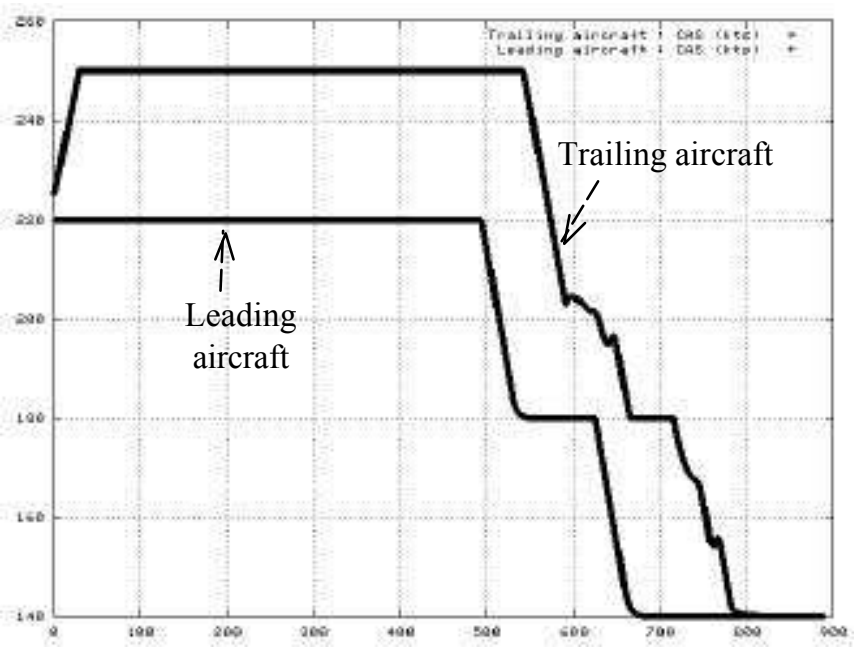

Figure 7: Leading aircraft and trailing aircraft indicated airspeed (kts) versus time (sec)

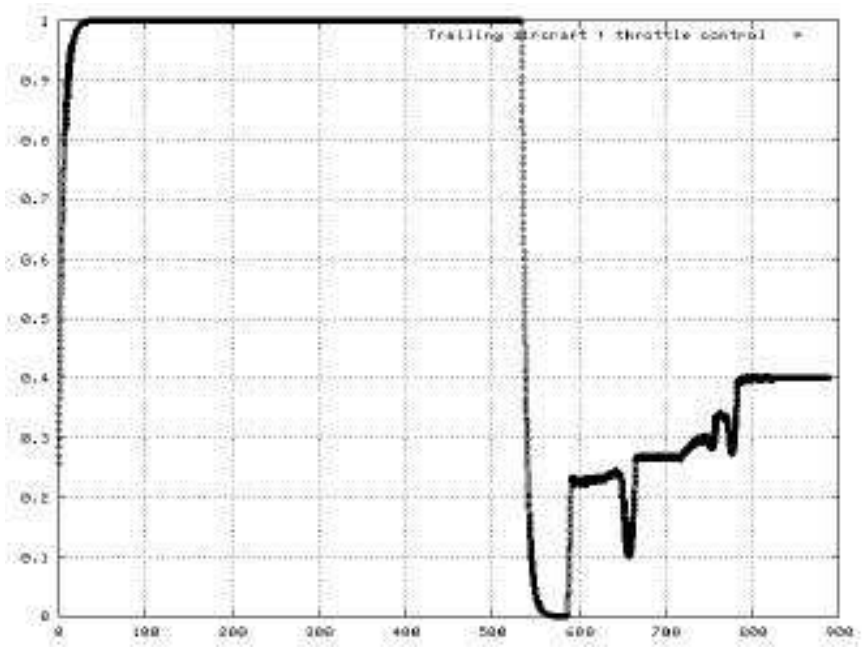

Figure 8: Throttle control versus time (sec) 
symmetric property of a matrix which appears in the relative motion equations. Furthermore, the design takes advantage of the Young's inequality to decrease the complexity of the controller due to the "explosion of terms" arising from the calculation of the control vector.

Simulation results based on a typical arrival procedure illustrates the efficiency of the proposed 'simplified' backstepping design. Nevertheless, it appears that the throttle control is quite demanding, which may induce an increase in fuel consumption. This deserves further studies in order to smooth this input as well as additional validation in terms of operational scenarios.

\section{Acknowledgments}

The authors wish to thank Philippe Louyot and Bernard Hasquenoph from the Centre d'Études de la Navigation Aérienne for their helpful inputs and comments.

\section{References}

1 European commission \& Eurocontrol, CARA/ASAS Activity 5 description of a first package of GS/AS applications, version 2.2, september 2002

2 Ivanescu D., Hoffman E., Zeghal K., Impact of ADS-B link characteristics on the performances of in-trail following aircraft, AIAA GNC Conference, Monterey, USA, August 2002

3 Agelii M., Olausson C., Flight deck simulations of station keeping, ATM 2001 R\&D seminar, Santa Fe, paper no. 17

4 Vanken P., Hoffman E., Zeghal K., Influence of speed and altitude profile on the dynamics of in-trail following aircraft, AIAA GNC Conference. Denver, Colorado, August 2000, Paper No. 2000-4362

5 Miquel T., Mora-Camino F., Levine J., Aircraft relative guidance: a flatness synthesis of a new autopilot mode , $5^{\text {th }}$ USA/Europe Air Traffic Management R\&D Seminar, Budapest, Hungary, June 2003

6 Miquel T., Mora-Camino F., Achaibou K., A feedback linearizing controller for relative guidance between commercial aircraft, AIAA GNC Conference. Austin, Texas, USA, August 2003, Paper No. 2003-5410

7 Hoffman E., Nanescu D., Shaw C., Zeghal K., Analysis of constant time delay airborne spacing between aircraft of mixed types in varying wind conditions, $5^{\text {th }}$ USA/Europe Air Traffic Management R\&D Seminar, Budapest, Hungary, June 2003

8 Krstic M., Kanellakopoulos I., Kokotovic P.V., Nonlinear and Adaptative Control Design, John Wiley \& Sons Ltd, New York, 1995

9 Nguyen X. Vinh, Flight mechanics of high performance aircraft, Cambridge University Press, 1995 